\title{
La adquisición de los términos cuantitativos en español. Estudio de un caso
}

\author{
FUENSANTA HERNÁNDEZ-PINA
}

Universidad de Murcia

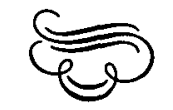

\section{Resumen}

El presente estudio intenta bacer una aportación en un área de innegable trascendencia para padres y educadores como es la incidencia del lenguaje incipiente en el aprendizaje de las matemá. ticas.

Los trabajos sobre este tema, realizados hasta la fecha, tanto en español como en otras lenguas, son relativamente escasos. No obstante, hemos podido observar cuatro bloques de investigaciones en función de los propósitos planteados.

Los objetivos de este trabajo son dobles. Por un lado, intentamos conocer la adquisición y secuencia de desarrollo de los términos cuantitativos en un niño hispanobablante. Por otro, establecer pautas provisionales para el desarrollo de los términos cuantitativos en español.

El sujeto de estudio fue el bijo de la autora, cuya evolución lingüística se siguió desde el nacimiento hasta los trea años cumplidos. El procedimiento para la recogida de datos consistió en grabación magnetofónica y anotaciones diarias de las emisiones lingüústicas libres. Los resultados muestran la amplia cobertura que presenta la cuantificación en el lenguaje del niño; desde el grupo de los cardinales, de uso frecuente, hasta el grupo de los cuantificadores, de pronta aparición. Destaca, igualmente, el nacimiento de los gradativos a partir de la igualdad.

Palabras clave: Adquisición del lenguaje, desarrollo del lenguaje, conceptos numéricos, conceptos matemáticos, conceptos cuantitativos, semántica, formación de conceptos, educación preescolar.

\section{The acquisition age of quantitative conceps in Spanish. . A case study}

\section{Abstract}

The aim of this artiche is to contribute to a field of undeniable importance to parents and educacionalists alike: the influence of developing language in the learning of mathematics.

Works in the subject are, relatively speaking, few and far between. Nevertheless, it has been possible to discern four main research programmes in this area.

This article serves a dual purpose. On the one band, an attempt is made to assess the acquisition and development of quantitatives in a Spanish-speaking child. On the other hand, some temporary guidelines are established for the development of quantitatives in Spanish.

The subject of this study was the author's son. His linguistic development from birth to the age of three was traced. In order to gather data use was made of tape-recordings and day-to-day annotations of free linguistic utterances. The results show the broad scope of quantification in the child's language, ranging from the set of cardinals (frecuently used) to the set of quantifiers (of an early appearence). To be noted also is the emergence of gradable word's on the basis of non-degree expressions.

Key words: Language acquisition, language development, number concepts, mathematical concepts, quantitative concepts, semantics, concept formation, preschool education.

Dirección del autor: Universidad de Murcia, Facultad de Filosofía y CC.EE. Departamento de Curriculum e Investigación Educativa.

Original recibido: Abril 1987. Revisión recibida: Marzo 1988. Aceptado: Mayo 1988. 


\section{INTRODUCCION}

La adquisición del lenguaje infantil es una materia por la que especialistas de varias ramas del saber muestran un gran interés. Parte de ese interés se ha venido polarizando en conocer las secuencias de adquisición lingüística longitudinalmente y ha nacido a raíz de lo hecho en otras lenguas. Estos estudios longitudinales nos están permitiendo conocer cuál es el proceso y los mecanismos de que se vale el niño independientemente de su entorno concreto. En español no son todavía muy abundantes las publicaciones de una y otra índole, aunque el tema está suscitando un interés creciente (Hernández-Pina (1979, 1981, 1984a, 1984b), Soto (1981), Siguán (1984), Vila (1985), Muñoz (1985), etc.). En este sentido, este trabajo forma parte de uno más amplio que quiere ser una aportación en un área de innegable transcendencia para padres y educadores: la incidencia del lenguaje incipiente en el aprendizaje de las matemáticas.

Partiendo de los datos abundantes que recogiera en su día para el estudio del habla de mi hijo Rafael (cf Hernández-Pina 1984a) me he propuesto abordar la adquisición de los términos cuantitativos en castellano. La secuencia de esta adquisición y su grado de extensión es uno de los aspectos menos estudiados en muchas lenguas, incluido el español. No obstante, a lo largo de los años 70 se interesan por el tema varios autores. Revisando la literatura pertinente observamos una cuadruple irrupción en el área. Por una parte, encontramos las investigaciones sobre la adquisición de los términos cuantitativos hasta más o menos la edad de 6 años o $1 .^{\circ}$ de EGB. Valgan por ejemplo Dutton (1963), Brace y Nelson (1965), Williams (1965), Donalson y Balfour (1968), Ginsberg (1971), Brush y otros (1978), etc. El objetivo primario de estos autores estriba en conocer los conceptos numéricos y cuantitativos que el niño tiene adquiridos a su entrada en el parvulario y buscar pistas para mejorar el currículo en aritmética a nivel de primaria.

El segundo grupo de trabajos se ha centrado en la elaboración de instrumentos para medir el grado de madurez que a nivel de cuantificadores alcanza el niño a la entrada del parvulario, puesto que se entiende que el desarrollo del lénguaje está íntimamente entrelazado con el desarrollo conceptual (Schwartz, 1969; Reys y Rea. 1970; Kraner, 1977).

El papel desempeñado por los padres en el desarrollo del lenguaje, sobre todo cuantificativo, ha sido objeto de análisis de un tercer grupo de estudios. Caso de Carron (1973), King (1984), etc. Y por último existen otros trabajos que tratan de contrastar las ideas de Piaget sobre el desarrollo del pensamiento cuantitativo: Estes (1959), Dodwell (1960), Elkind (1961).

Como es sabido, Piaget (1952), ha sido quien mejor ha estudiado la evolución y desarrollo del pensamiento abstracto en el niño, que él sitúa en etapas progresivas marcadas por límite de edad bastante precisos. Así, en la etapa primera (la sensoriomotora), que abarca hasta los dos años, el niño siente y explora el mundo circundante pasando de un uso indiscriminado de los objetos hasta el uso discriminado de los mismos. Los objetos en esta etapa son algo exterior, permanentes a pesar de sus posiciones, y poseedores de unos atributos discriminativos que paulatinamente el niño descubrirá en su constante interacción con los mismos. Pero inicialmente se contentará con la simple manipulación de tales objetos sin interesarse por los atributos específicos.

Al rebasar los dos años entramos en la etapa del pensamiento simbólico (Piaget e Inhelder, 1969). El niño comienza a atribuir funciones simbólicas propias 
de los objetos a los que unirá el simbolismo del lenguaje, con la particularidad de que este último por ser de naturaleza social le impondrá, pese a su carácter convencional y arbitrario, unas pautas en gran medida prefijadas. Sin embargo, en esta etapa, que abarca de los dos a los cuatro años, los conceptos lingüísticos del niño serán tan volubles y flexibles como las funciones que él atribuye a los objetos.

Pero gracias a la interacción social, el lenguaje irá ganando terreno inexorablemente, lo que comportará a la postre una fuerte reestructuración de la función simbólica idiosincrática del niño.

No es mi propósito, ni mi competencia, analizar críticamente el desarrollo del pensamiento cuantitativo sobre las pautas del ilustre psicólogo suizo. Pretendo, más bien, concentrarme en los estadios incipientes del lenguaje cuantitativo, en línea con los trabajos de tipo empírico antes mencionados, concretamente con los primeros categorizados. Sin embargo, a diferencia de estos trabajos, pretendo cubrir de 0 a tres años y medio (todos ellos parten de los tres años más o menos) con el fin de ver el nacimiento de los cuantificadores. También, a diferencia de los autores citados (todos ellos extranjeros), creo oportuno establecer un marco lingüístico de cuantificación del adulto lo más completo posible (no desarrollado ni en inglés ni en español, que sepamos). Sólo partiendo de dicho marco podemos llegar a ver con nitidez la evolución de los cuantitativos en español. A partir de este planteamiento será factible llegar a elaborar un trabajo comparativo con otras lenguas, todavía inexistente. Espero, por otra parte, que dicho marco sirva de pauta a quienes se interesen por profundizar en este aspecto con niños hispanohablantes.

\section{Definición de cuantitativos}

Unas de las opciones binarias semánticas operativas en la lengua que afecta a la oración es la de cuantificación (otras posibles son la determinación, la deíctica, la negación, la posesión, el énfasis prosódico, la pasivización, etc.) La cuantificación supone poner de relieve un determinado grado de cantidad, que abarca desde la inclusión total hasta la ausencia de dicha cantidad. Se trata por tanto de una escala que corta a través diversas partes de la oración afectándola de modo diverso.

En el caso de los sustantivos, por ejemplo, el parámetro cuantificación los divide en dos grandes grupos: los contables, susceptibles de ser matizados cuantitativamente a partir de la división básica de número (ejemplo: algún libro, la mitad de la lección, tres novelas, muchos coches, etc.), y el grupo de los in-. contables o nombres masa; que rechaza en general por su misma esencia la categoría de número, pero sí admiten cuantificación (ej. todo el dinero, bastante agua, menos color, etc.). Decimos «en general» y no «siempre», porque también. encontramos híbridos que siendo incontables pueden pasar a ser contables (ej. un té, dos tes = dos tazas de té). Sin embargo, esta peculiaridad no altera la. esencialidad de la dicotomía establecida que se pone de manifiesto en los siguientes rasgos estructurales:

a) Los sustantivos contables, a diferencia de los incontables/masa admiten. cuantificación numérica, ya se trate de cardinales (ej. cinco árboles), ordinales (ej. la segunda calle), o colectivos (ambos lados, un par de botas, etc.). En cambio, los incontables pueden admitir, junto con los contables, una cuantifica- 
ción numeral partitiva (ej. una décima parte del dinero/de los libros) o multiplicativa (ej. el triple del dinero/de los libros).

b) Los sustantivos incontables explican el nivel cuantitativo mediante la anteposición de lexías que en número mucho más reducido que los cuantificadores numéricos abarcan como aquellos desde el 0 (nada o cuantificación nula) al todo. Estos cuantificadores que podríamos denominar no específicos tiene un comportamiento morfológico distinto según acompañen a sustantivos contables o no. Con los contables, y por razones obvias, no toman normalmente la forma plural (ej. mucho dinero, poca arcilla), mientras que con los contables puede darse el singular y el plural (ej. este árbol tiene pocas hojas/poca hoja).

No todas las partículas cuantitativas sirven indistintamente para acompañar a los contables y a los incontables: algunos cuantificadores no específicos no ocurren en uno u otro grupo. Concretamente varios acompaña exclusivamente a sustantivos contables; algo solo ocurre con incontables, lo mismo que nada, etc. (cf. Tabla I).

\section{TABLA I}

Cuantificadores sustantivos

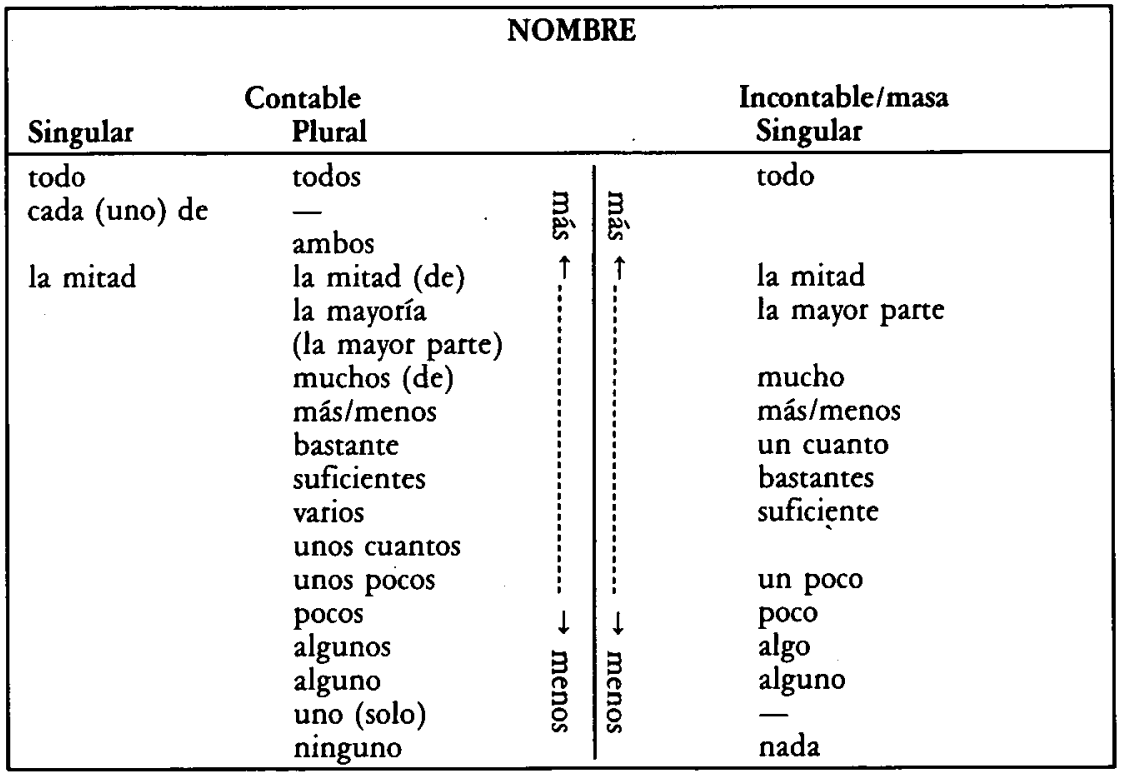

c) Existe un tercer grupo de gradativos, numéricamente menor que el de los no específicos, que afecta no sólo al sustantivo (ya sea contable o no), sino que también lo hace al adjetivo y al adverbio. Me refiero a los cuantitativos gradativos constituidos por las partículas que marcan los tres grados del comparativo (más/menos... que), las formas de igualdad (igual... que; tan + adverbio/adjetivo... que/como; tanto/a + sustantivo... que/como) (ej. hay tanta leche como en...; es tan alto que... etc. así como el superlativo (es el libro más caro de la tienda, es el nieto más nieto de todos los nietos, etc.).

d) También afecta la cuantificación a los pronombres, en los que es preciso distinguir dos grupos: aquellos que van seguidos de la partícula $\mathrm{DE}$ y otros que no llevan dicha partícula. Los primeros son cuantificadores partitivos (del tipo alguno de ellos, muchos de sus libros, cada uno de nosotros, varios de ellos, 
etc.) que hacen referencia, como hemos visto al hablar de los cuantificadores numéricos y no específicos, a sustantivos contables. No obstante, también pueden tomar la partícula de los cuantificadores que preceden a sustantivos incontables (un poco de dinero, nada de agua, etc.) y el artículo determinado (alguno de los albañiles, muchos de los visitantes, etc.). Dentro todavía de los pronombres cabe distinguir entre los «indefinidos cuantificadores que hacen referencia personal y los que abarcan desde la total presencia a la ausencia, tal como recoge la tabla II, con referencia no-personal.

TABLA II

Pronombres cuantificadores impersonales

\begin{tabular}{|ll|}
\hline Referencia personal & Referencia no personal \\
\hline Todos están aquí & Lo he hecho todo \\
Algunos vinieron ayer & Lo he hecho casi todo \\
Alguien llama & Tengo algunos (libros) \\
Casi nadielcasi ninguno vino & Algo pasa \\
Nadie/ni uno apareció por allí & No tengo casi nada \\
& No tengo nadalni un(a) \\
\hline
\end{tabular}

${ }^{1}$ Para mejor comprensión del lector transcribo las frases en lenguaje adulto. Naturalmente, el niño no las pronunciaba así en esta etapa. Para más detalles of. Hernández-Pina (1984a).

e) El últimọ grupo de cuantificadores lo constituye términos graduables pertenecientes todos ellos a la categoría gramatical del adverbio que, como es sabido, se caracteriza por una gran hetereogeneidad. La cuantificación en este caso afecta a tres niveles: el de frecuencia, el de duración y el de grado. La frecuencia se mueve entre los límites del siempre y el nunca, mientras que la duración lo hace entre el siempre y el nada; y el grado, a su vez, oscila entre el total/enteramente y la negación absoluta de grado.

\section{OBJETIVOS}

Dos son los objetivos que persigo con este trabajo. Por una parte, conocer la adquisición y secuencia de desarrollo de los términos cuantitativos en un niño hispanohablante. Trato, en segundo lugar, de establecer pautas provisionales para el desarrollo de los términos cuantitativos en español.

\section{METODO}

\section{Sujetos}

El sujeto de este estudio ha sido mi propio hijo, cuya evolución lingüística he seguido desde el nacimiento hasta la edad de tres años y medio. El niño es hablante monolingüe de español presentando un desarrollo psicológico normal. Durante el período de recogida de datos estuvo básicamente en contacto con el habla de sus padres, ambos universitarios. 


\section{Procedimiento}

El procedimiento utilizado para la recogida de datos consistió en grabaciones y anotaciones diarias de las emisiones lingüísticas libres del sujeto de estudio desde el nacimiento hasta la edad de tres años y medio. En el caso de las anotaciones siempre se indicó el contexto situacional, dato imprescindible para ulteriormente interpretar correctamente emisiones lingüísticas polivalentes o anfibológicas. A partir de ese corpus hemos extraído todas las emisiones que contenían términos cuantitativos, base de este estudio.

\section{ANALISIS DE LOS DATOS}

De acuerdo con el esquema presentado en la introducción, analizaremos la adquisición de los cuantificadores en el sujeto de estudio comenzando por los cuantificadores numéricos.

\section{a) Cuantificadores numéricos}

El nombre y las frases nominales son el medio a través del cual la gramática organiza la forma en que nos referimos a los objetos. Como he indicado en otro trabajo (Hernández-Pina, 1984a) el sustantivo es la primera clase gramatical representada en el habla del niño. Tanto en la etapa holofrástica como en la de dos palabras, la inmensa mayoría de los nombres pertenecen al grupo de los concretos, con un reparto entre contables e incontables favorable al grupo primero, aunque los ejemplos de los segundos también se prodigaron.

En la distinción del plural frente el singular creemos estriba el comienzo de la cuantificación en el niño, puesto que supone la realización de la pluralidad de objetos frente a la simple unidad. Ello explica, creo, la casi ausencia de sustantivos plurales en los estadios iniciales de la etapa holofrástica. El primer atisbo del artículo, que apareció formando bloque con el núcleo nominal, será una prueba evidente del comienzo del proceso por el cual el nombre generalizado irá ganando paulatinamente en concreción para, posteriormente, marcar una primera y fundamental diferencia con la pluralización. Esto se pondría más claramente de manifiesto en la fase de dos palabras, cuando el artículo aparece claramente deslindado del elemento nominal. El singular sería todavía la tónica dominante desde los 18-24 meses, de tal modo que casi un ochenta por ciento de las emisiones se caracterizaron por la singularidad. Sin embargo, y puesto que se trata de un proceso evolutivo constante, ya desde los 18 meses harían aparición formas esporádicas de plural que ganarían en frecuencia y uso hasta llegar a su dominio casi pleno hacia los 26 meses. (Hernández-Pina, 1984:238).

Para esta edad la experiencia almacenada de los meses anteriores resultará imprescindible en el desarrollo del pensamiento abstracto; junto al conocimiento de los objetos que manipula, el niño comenzará a utilizar las palabras que representan a esos objetos experienciados, dando lugar de este modo al uso de los términos cuantitativos numerales. Para poder contar el niño no necesita conocer los nombres de todos los números; basta simplemente con conocer los primeros dígitos, aunque hay que precisar que se dan dos niveles de uso: uno mecánico y otro racional. El primero servirá de paso hacia el segundo. De todos es conocido que a los niños de corta edad les encanta recitar mecánicamente los primeros diez dígitos. En el parvulario se suelen enseñar rimas ${ }^{1}$ que con- 
tienen los diez primeros números para de este modo poder dominar los términos y pasar luego a introducir su significado. En la escuela, una vez dominados tales números, se potenciará un aprendizaje numeral de los dígitos superiores eminentemente mecánico.

\section{- Los numerales}

En el caso que nos ocupa los numerales no surgirían a nivel activo hasta avanzada la etapa de las dos palabras. Nada detecté en la etapa holofrástica que indicase un uso mecánico, ni menos aún racional, de los números. Sin embargo a partir de los 23 meses los numerales cardinales (las otras categorías numerales son más tardías) irrumpen en el lenguaje del niño un tanto mecánicamente:

- cuatro gatos (no eran cuatro) 23 meses

- cuatro niños (idem) 24 meses

- siete kilos y media (dicho sin sentido) 25 meses

- mamá, habla 1, 2, 3, 4, 5, 6... 28 meses

- mamá, ya sabe el nene 1, 2, 3, 4, 5, 6... 29 meses

Quisiera resaltar lo de «un tanto mecánicamente» porque no estoy segura de si en la conciencia de mi hijo existía el concepto de cuantificación de modo subyacente. Es muy probable que los números empezaran ya a indicar un grado de cuantificación todavía poco preciso, pero real. La adquisición racional del uno, con su doble acepción de numeral cardinal y artículo indefinido, se hizo patente de los 24 a los 26 meses (un ratón, 24 meses; una farola, 25 meses; papá ca(ra)melo, uno solo, 25 meses). El paso siguiente, el desdoblamiento de la unidad sea fraccionaria o aditivamente, ocurrió por la misma fecha, y no ya mecánicamente sino racionalmente, como veremos en seguida.

Según Skemp (1971) los conceptos se construyen jerárquicamente. A partir de nuestra experiencia sensorial formaríamos unos conceptos primarios, dependientes exclusivamente de dicha experiencia, y otros secundarios, cuya aparición estaría supeditada a los primarios. Así, el concepto de dos no se formaría en tanto el niño no haya internalizado la unidad y descubierto la similitud de dos unidades. La idea numérica racionalmente asimilada (el 4, el 8, el 10, etc.) sería un concepto terciario; la suma sería un concepto de cuarto orden, etc. Es decir, la cuantificación numérica es para Skemp una jerarquía de conceptos que va de lo general a lo particular mediante estadios fijos semejantes a la secuencia inmutable de desarrollo piagetiana.

Pese a lo atractivo de la hipótesis, mi opinión, a la vista de los datos de Rafael, difiere de la de Skemp en lo referente a la idea de «similitud» de dos unidades. El concepto dos, al menos en mi caso de estudio, se alcanzó previo al dominio

a. de los sustantivos contables

b. del concepto otro, enormemente vinculado no ya al grado de semejanza (como apunta Skemp), sino al de identidad (= igual).

Realmente la idea de identidad tuvo un triple reflejo lingüístico representado por las voces:

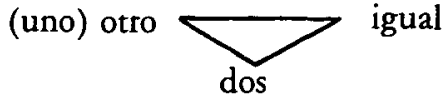


que tuvieron un uso inicial indiscriminado. Los ejemplos son, creo, elocuentes ${ }^{2}$ :

- otro bus

- otra sartén

- otra igual

25 meses

- igal payaso

- igual coche

- igual carrito

25 meses 0

- dos colmillos

- había dos autobuses

- dos tigres

25 meses

- dice papá que es una peonza y dos peonzas, una peonza y otra peonza (32 meses).

No obstante, a los 30 meses empieza el deslinde progresivo de las tres partículas mencionadas, proceso que culminará hacia los 34 meses. El dos empezará a surgir como algo no necesariamente vinculado al concepto de igualdad y por primera vez aplicado a sustantivos contables, no ya concretos sino abstractos. Ejemplo:

- es igual los dos - 32 meses

- me ha cuentado (contado) dos cuentos papá - 32 meses

- mira, dos autobuses, uno verde y otro rojo - 33 meses

- mira, dos autobuses, uno grande y otro pequeño - 33 meses

- mira, dos iguales (para-choques) - 32 meses

A los 34 meses, pues, aparecía el numeral dos plenamente adquirido frente a los otros dos miembros de la terna. Otro, por su parte era usado tanto estructural como semánticamente, como en el lenguaje adulto. En cambio, igual, si bien apareció con una semántica clara, presentaría variaciones estructurales en conflicto con como hasta bastante tardíamente. Ejemplo: aqui bay dos volantes como iguales (33 meses). La variante colectiva de dos (i.e. par) la utilizó nuestro informante por primera vez a los 40 meses: jandá qué par de autobuses!.

El numeral tres hizo su aparición a los 37 meses (ejemplo: tres libros) pero no presentó características de adquisición plena hasta los 43-44 meses. Ejemplos:

- nos queremos mucho los tres ( 43 meses)

- tenemos tres tubos: uno para la comida, otro para el pipí, y otro para la caca (44 meses).

Cabe resaltar el paralelismo existente entre edad biológica y la adquisición. de los números cardinales. Ello explica tal vez la ausencia de otros dígitos en la etapa que me ocupa.

Del resto de los numerales no hay mucho que decir, puesto que tienen un componente formal que los aleja del lenguaje cotidiano, salvo algunos casos dignos de mención.

De la serie de los ordinales sólo constatamos el uso de primero, que surge a los 28 meses con un doble matiz. En solitario, indicando orden:

- primero, el mes - 28 meses

- no, pipí, primero (al mandarlo a la cama) - 28 meses. En paralelo, seguido por adverbios indicadores del orden preferencial:

- primero oso, ahora elefante - 28 meses

- primero el otro y luego el uno - 35 meses

- primero cogemos todos y luego pasamos la aspiradora - 35 meses

De los distributivos, solamente cada uno tuvo un uso regular a partir de los 
31 meses, fecha de su aparición, no exenta de alguna contaminación por analogía. Primeramente su uso fue correcto (ejemplo: uno cada uno - 31 meses). Más tarde encontramos casos del tipo: cada uno y cada otro ( 35 meses), de uso esporádico. No registramos ningún multiplicador y la serie de los partitivos tampoco tuvo incidencia especial, puesto que los numerales pueden suplirlos en muchas ocasiones. Nuestro informante recurrió a los cardinales -fundamentalmente al dos- para dar el matiz partitivo:

- un niño partió el caramelo en dos (por la mitad) - 37 meses

- se rompió el vagón en dos (por la mitad) - 44 meses.

\section{b) Cuantificadores no específicos}

Decíamos más arriba, al hablar de los cuantitativos, que existía un número menor de numerales que abarcaba del todo al nada y que afectaba a sustantivos tanto contables como incontables. Decíamos también que alguna de estas lexías es bidireccional frente a otras que son solamente unidireccionales. Esto en el habla del adulto.

En el habla del niño objeto de análisis, el nacimiento de los cuantificativos no específicos se produjo a partir del uso activo de la partícula MAS. Este será por tanto el primer término del grupo, que irrumpiría desde la suprageneralización semántica (cf. Bloom, 1973). Por una parte serviría a los 17 meses como elemento afirmativo-confirmativo en oposición a no (F. Hernández-Pina, 1979: 223); así a la pregunta ¿quieres más pan?, la respuesta durante la etapa de las dos palabras fue normalmente más, en lugar de sí, seguido opcionalmente por un sustantivo (galletas, pan, etc.). Más sirvió, igualmente, para pedir la repetición de un acontecimiento, señalar una cualidad o un objeto: canta más, otra más, trae más, etc. Sería el más equivalente a «otra vez» o iterativo. Junto a estos dos usos aparece la cuantificación; primeramente (hacia los 21 meses) para indicar carencia aunque desprovista la partícula de la negación correspondiente (i.e. más = «no hay más»), para muy pronto - hacia los 23 meses- consolidar su posición tanto semántica como estructuralmente:

- más agua) (19 meses)

- más música (23 meses)

- más moras (25 meses)

Tenemos por tanto que para los 23-24 meses figura esta lexía en el elenco de Rafael con los valores semánticos que recoge la figura:

$$
\text { MAS }\left\{\begin{array}{l}
=\text { asís (ej. ¿Quieres más pan? - Más. } \\
=\text { «otra vez» (ej. Canta más) } \\
=\text { cantidad }\left\{\begin{array}{l}
- \text { ausencia (ej. más }=\text { no hay más) } \\
- \text { presencia (ej. más agua) }
\end{array}\right.
\end{array}\right.
$$

Alcanzado este estadio, no es de extrañar que el niño comenzase a ampliar la cobertura cuantitativa de la partícula en el terreno de los agradativoss. Antes, no obstante, aparece el más que podemos llamar «ponderativo» (ej. iQué torre más grande!, ¡Qué tío más gordo, etc. - 27 meses), precedido por el más en función adverbial (ej. más hay - 23 meses), Ambos usos aparecen consolidados para los 28-29 meses:

- Mamá, no riñas más al nene (28 meses)

- Mamá, no duermas más (idem)

- Mamá, abre la puerta; abre más (32 meses). 
Este último ejemplo da pie para hacer un breve comentario sobre la no existencia en el corpus del aparente correlato de más: menos. En el lenguaje adulto damos por sentado que ambos gradativos son imprescindibles para indicar un grado de cuantificación mayor o menor. Sin embargo, no parece ser así realmente; o al menos no necesariamente. En frases ponderativas como las que hemos visto u otras similares, el uso de menos queda descartado por mostrar la lengua preferente por opuestos nominales que siguen a más. Es decir, el hablar adulto dirá:

$$
\text { - Qué casa más }\left\{\begin{array}{l}
\text { grande } \\
\text { pequeña }
\end{array}\right.
$$

pero no dirá: - Qué casa menos $\left\{\begin{array}{l}\text { grande } \\ \text { pequeña }\end{array}\right.$

El más en estos casos entra en sinonimia con tan (que no utilizó mi hijo): ¡Qué casa tan grande/pequeña!

En usos no ponderativos el hablante puede utilizar la partícula menos, pero puede igualmente recurrir a otras formas. Por ejemplo, a la pregunta ¿Quieres más? la respuesta atenuativa admite expresiones como: no (más), no tanto, es mucho, la mitad, y - por supuesto- menos. De ahí que no me sorprenda que no figurase esta lexía en el corpus del niño. Las equivalencias que él utilizó en la edad que nos ocupa fueron:

NO MAS: Mamá, no duermas más (duerme menos) - 29 meses

NO: ¿Quieres más agua? - No.

SOLO UN POQUITO: Y más no; sólo un poquito (32 meses).

El otro gran eje cuantitativo con marcadores no específicos abarca desde la plena cuantificación a la cuantificación nula con un margen amplio en el centro (el algo) que el hablante cubre en mayor o menor grado. En nuestro caso emergió en primer lugar la cuantificación total a los 22 meses, consolidándose semánticamente a los 25 , si bien se detectaron fluctuaciones a nivel estructural hasta los 32-33 meses:

a. Todos (reclamando las piezas del lego)

- 22 meses

b. Todos queman (= todas las tazas de té queman)

- 23 meses

c. Todos pinzas ( = todas son pinzas)

- 24 meses

d. A todos a mesa (= todos a la mesa)

-25 meses

e. Todo perros ( $=$ todos son perros)

- 26 meses

f. (Es)tudiando todos

- 27 meses

g. Me ha tirado todo el nene (= me he tirado entero al suelo) - 29 meses

h. Se ha ido la toda agua (de la bañera)

i. Papá me ha curado todo

j. Ya está todo limpio

- 31 meses

- 32 meses

-33 meses

Inicialmente el niño adquirió el uso de todo(s) con contables (expresos o sobreentendidos), apareciendo los incontables a los 32-33 meses (cf. ej. $i, j$. Precisamente coincide este uso con la adquisición de cuántos, la partícula genérica cuantitativa por excelencia, necesaria para indagar sobre cualquier grado cuantitativo (ej. ¡Cuántos libros que hay aquí! - 32 meses). Creo que esto es un claro indicio de la adquisición de la cuantificación en sus líneas esenciales. Hasta esta fecha el niño había utilizado la expresión qué muchola(s): ¡Mamá, qué mucha! (agua) = cuánta agua. La no cuantificación o cuantificación nula es anterior, sin embargo, a los dos años. Atrás mencionaba que más presentaba en uno de sus usos un claro matiz de ausencia cuantitativa evidente en el niño a los 19 
meses. Con la aparición de $N O$ a los 20 meses tendremos el elemento fundamental de cuantificación nula (ej. no caca, no papas, - 20.21 meses respectivamente). Casi de inmediato irrumpiría la forma nada, primero en solitario y enseguida como partícula reforzativa de la negación:

- Abre boca nada (= no hay nada en la boca) - 22 meses

- Aquí no hay nada - 26.27 meses

- No tengo bici ni nada - 28 meses

A los 30 meses la dicotomía todo-nada estaba plenamente adquirida:

- No hay nada. Ha comido nene todo (30 meses).

Un hecho que nos llamó la atención, y que precisa confirmación por otros estudios, fue el modo de adquisición de la cuantificación intermedia, equidistante entre el atodo» y el «nadas. Entre los 31.32 meses registramos varias frases en las cuales el «algo» cuantificable se manifestó por medio de la lexía nada:

1. Niño: ¿Dice nada Rafaelín? (= Dice algo?)

Madre: No, no dice nada

Niño: Sí dice nada Rafaelín (32 meses)

2. Madre: acércate, que no hace nada la aspiradora

niño: sí hace nada la aspiradora - 32 meses.

Esta suprageneralización puede deberse, pienso, a dos causas: por un lado, el estímulo adulto era mayor en la cuantificación nada (no hace nada, no hagas nada, nada de nada, ni nada, etc.), y así se pone de manifiesto en los dos ejemplos citados; por otra parte existe en el lenguaje adulto una aparente sinonimia entre algo-nada en algunos casos de uso frecuente que bien pudiera arrastrar por analogía el niño a otros en los que tal acontecer no existe. Piénsese por ejemplo en: ¿no dices nada? emitido por el niño a los 31 meses que equivale semánticamente a ino dices algo?

A partir de los 32 meses irrumpe algo de un modo explícito y perfectamente deslindado:

- ¿se ha caído algo? - 32-33 meses

- quiero a ver si hay algo dentro (de la caja) - 34 meses

- papá, dí algo de mi - 34 meses.

El par ninguno-alguno y sus correlatos nadie-alguien aparecen más tarde que el anterior. Hacia los 30 meses el primero en surgir sería nadie, al que seguiría ninguno, alguien y alguno por este orden. Ejemplos:

- pato feo no quiere nadie (28 meses)

- yo estoy descalzo como Tomasito, pomo los zapatos no me pinche ningún pie (32 meses)

- ¿ha venido alguien? (35 meses)

- ¿no está alguno? (37 meses) (= no hay ninguno).

Los usos de los dos últimos, sin embargo, fueron muy escasos y como refleja el último ejemplo todavía inestables.

Otro par de cuantificadores intermedios adquiridos por el niño hasta los tres años y medio fue mucho(s)-poco(s). Lo primero que llamó nuestra atención fue la abundancia de ejemplos del primero frente a la escasez de los referentes al segundo. Como en el caso de todo(s), aparece mucho(s) con contables y prácticamente a la vez que el cuantitativo total (a los 23 meses). Ejemplos:

- muchas silletas (23 meses)

- papá tiene mucbo pelo (26 meses)

- mamá, hay muchas montañas (27 meses). 
Ya dejamos indicado que inicialmente muchos fue el genérico utilizado por Rafael para referirse a la cuantificación (= cuántos!)

El uso de mucho con incontables aparece a los 26 meses y se consolida rápidamente. Ejemplos:

- me quiere mucho (ele)fante (25 meses)

- pesa mucho nene (26 meses)

- mucho (respuesta a la pregunta ¿te gustan las papas = comida (28 meses)

- be bebido agua, mucha agua (31 meses)

- Rita no pegues nene, po(r) que baces daño, mucbo daño (28 meses).

Junto con mucho(s) también detectamos la forma indefinida un montón (ejemplo: abi tienes un montón de libros (33 meses), yo tengo abi muchos, un montón (33 meses).

En cuanto a poco(s) hizo su aparición en forma diminutiva a los 31 meses y aparece usado con pleno dominio incluso con incontables. Ejemplos:

- ¿la apago un poquito la luz? (31 meses)

- no bay poco, hay muchos (libros) (31 meses)

- no hay poco, hay mucho (yogur) (32 meses).

El último cuantificador intermedio que aparece en el corpus de Rafael fue bastante usado como incontable. Ejemplo: con ese tengo bastante (papel) (30 meses).

\section{c) Cuantitativos gradativos}

La adquisición de los cuantitativos gradativos tuvo lugar a partir de las formas de igualdad. Tal como señalábamos al hablar de los numerales, el concepto de igualdad nace a los 22 meses. Primero de una manera discreta para posteriormente establecer una gradación idéntica entre dos objetos. Así, a las secuencias iniciales del tipo igual payaso, letras iguales, igual cayó, otro igual (25 meses) en que solamente se ponía de manifiesto la semejanza del objeto, aparece a los 25 meses también el grado central de comparación: el comparativo de igualdad (ejemplo: igual e jaca = igual que la jaca). De los 26 a los 35 meses utilizó nuestro informante la alternativa con como (X es como $\mathrm{Y}$ ). Precisamente por la identidad semántica de ambas estructuras observamos en el período que comentamos constantes contaminaciones en los ejemplos, tanto en los referentes a cuantificación individual (ejemplo, es como igual - 34 meses, ya señalado al hablar anteriormente), como en las comparaciones de dos objetos (ejemplos: mira, iguales así como esto - 27 meses; mira, reloj igual como ese - 28 meses; es una guitarra como igual de papá - 34 meses; es como igual como yo - 41 meses), lo cual no ha de interpretarse como que el niño cometiese errores comprensibles de modo sistemático. A los 38 meses, por ejemplo, me decía: cuando un niño se pone grande como su papá le compra unos patines. En este ejemplo se patentiza la adquisición simultánea de la cuantificación para expresar «tamaño». Otros ejemplos en este sentido son:

- póntete a ver si eres de grande como el árbol de Navidad - 41 meses

- cuanto más grande más duro se pone (refiriéndose a una carpeta con libros).

Para los 43 meses todos los ejemplos que encontramos en el elenco de datos reflejaron un dominio adulto del grado comparativo de igualdad, ya se tratara de la construcción con igual o de la construcción con como. Ejemplos:

- éste no es como éste, éste es igual que éste (refiriéndose a unos osos de peluche). 
Kraner 1977 establece de los cuatro a los seis años la edad de la adquisición de la igualdad cuantitativa. Por los datos expuestos (susceptibles de confirmación por otros estudios sobre el español) el niño adquiere el concepto de igualdad entendida como identidad plena antes incluso de cumplir los tres años de edad. En cambio, la igualdad referida a un aspecto de los varios susceptibles de comparación (tipo: es tan alto/blanco etc... como) no apareció en nuestro sujeto en el período que nos ocupa. Los gradativos para indicar cuantificación en mayor o menor escala entre dos objetos (tipo más... que/menos... que) no se consolida hasta los 45 meses de edad aunque encontramos algún ejemplo previamente:

- la torre es alta que mi - 39 meses

- he comido más que tú - 45 meses

- tengo más que tú - 45 meses

Se observa en ellos la ausencia de gradativos/comparativos de inferioridad, lo que atribuyo, al igual que viéramos al hablar de la pareja más-menos, a la menor funcionalidad de menos, que puede ser reemplazada por otros atenuativos. En Rafael encontramos únicamente el ejemplo siguiente: abora nos queda poco que antes (menos que) 35 meses.

En cambio, las formas superlativas fueron bastante abundantes. Desde los 29 meses observamos una utilización del cuantitativo muy reemplazado esporádicamente en las etapas iniciales por mucho. Ejemplos:

- es muy alto autobús - 28 meses

- hace mucho oscuro (muy) - 29 meses

- hace mucho de noche (muy) - 29 meses

- es grande, mucho grande (muy) - 33 meses

La explicación de esta actuación podría venir dada en términos conductistas. El niño oía como respuesta a preguntas con muy adjetivo/adverbio una confirmación con la partícula mucho (¿es muy alto? respuesta: mucho). Un defensor del innatismo tendría aquí un claro ejemplo, sin embargo, de generalización por analogía: si hace mucho frío es lógico que haga mucho de noche o mucho oscuro. Me inclino, personalmente por la segunda explicación como más plausible, aunque no descarto plenamente la primera. En cualquier caso me interesa resaltar que por una u otra vía el niño llegó a afianzar la forma correcta hacia los 40 meses. Ejemplos:

- dame los alicates para cortar la púa, que está muy largo - 39 meses

- mañana hay que comprar un elefante muy grande -40 meses.

Las formas en -ísimo no fueron muy socorridas, pero tuvieron una aparición regular a partir de los 30 meses. Así registramos las siguientes expresiones:

- es un montón grandísimo - 30 meses

- qué casa más grandísima - 31 meses

- jandá! qué uno más grandísimo - 31 meses

- yo tengo dos (ha)bitaciones grandisimas - 34 meses

En cambio sí fueron frecuentes las formas de superlativo que pudieramos llamar aperifrásticas», tales como:

- casita grande grande - 25 meses

- babia un tren largo de mucbos trenes - 30 meses

- jandá! qué tortilla más grandona - 37 meses

El superlativo reduplicativo (grande grande) no tuvo contrapartida formal al no hablar de los términos opuestos (pequeño frente a grande, bajo frente a al- 
to, etc.) En estos casos nuestro informante recurrió sistemáticamente al uso de formas diminutivas.

Ejemplos:

- mamá, mira caja pequeñita (26 meses)

- dónde (es)tá libro pequeñin (28 meses)

- avión pequeñito tiene alas grandes grandes (28 meses)

- un puente pequeñito y un puente grande (32 meses)

Fue menos usual la perífrasis con asi de. Ejemplo: ha pusido un árbol de navidad asi de pequeño. (41 meses).

La cuantificación puede también darse en el idioma en términos de frecuencia, que puede ser definida (ejemplo, 3 veces) o indefinida (ejemplo, a menudo). Ambos tipos aparecen en el corpus entre los 30 y 35 meses de edad, aunque en sus líneas más esenciales. Así, en la frecuencia indefinida los ejemplos se redujeron al uso de siempre y nunca, sin matizaciones intermedias. Ejemplos:

- esta caja está vacía siempre (35 meses)

- no, nunca más (35 meses) (respuesta a mi pregunta: no me harás daño ¿verdad?)

En el caso de frecuencia definida abundaron más los ejemplos; sobre todo los de numeral + vez:

- yo lo ba enseñado una vez a la maestra (35 meses)

- la apago y la enciendo otra vez (la luz) (35 meses)

- pero yo se lo ha enseñado 3 veces (el dibujo a su tío) (35 meses)

- mamá, te he dicho muchas veces que este jersey tienes que tirarlo a la basura ( 43 meses)

Los que hacen referencia a la cuantificación (apartado d) no tuvieron reflejo alguno en la etapa que nos concierne. Ya mencionamos los distintos cuantificadores de grado utilizados por nuestro informante en relación con los apartados c y e. Los que hacen referencia a la duración (apartado e) no hicieron su aparición (consecuencias temporales, claro está) durante la etapa que hemos analizado, lo cual corrobora otras investigaciones que señalan la adquisición de los esquemas temporales bastante más tardíamente; de 5 a 8 años.

\section{CONCLUSIONES}

Por lo visto en las páginas anteriores cabe hacer varias observaciones significativas. La amplia cobertura que presenta la cuantificación en el habla adulta encuentra en el lenguaje incipiente unos ecos muy tempranos a partir de estrategias muy claras. Así en la cuantificación numéricas destaca por su frecuencia y uso el grupo de los cardinales y, en efecto, nuestros datos patentizan esa prelación que partiendo del todo indiviso va paulatinamente diversificándose en sorprendente coincidencia con la edad biológica. Este nivel básico servirá para el asentamiento del resto de las estructuras numéricas fundamentales a la entrada del niño en la escuela.

En el grupo de los cuantificadores no específicos destaca la pronta y segura adquisición de más frente a la ausencia de menos, lo que de ser generalizado corrobora la práctica ancestral de iniciar las matemáticas por el aprendizaje de los procesos aditivos y no de los sustractivos. Observamos, igualmente, que se da un adelanto adquisitivo de los cuantitativos positivos frente a los negativos: además del ejemplo de más-menos, se hizo esto patente en pares como todo- 
nada, mucho(s)-poco(s), etc. En relación con esto entiendo a la vista de los procesos observados que el niño adquiere primero la cuantificación con contables, para más adelante abstraer el proceso y aplicarlo a no contables o nombres masa.

Finalmente, es de notar el nacimiento de los gradativos comparativos que a partir de la igualdad, sinónimo inicial de identidad, el niño ha de ampliar, primeramente hacia la maximización (más... que) o cuantificación en términos muy notorios (muy... o -ísimo/a) para, por último, dominar la minimización. Según nuestros datos, para los tres años y ocho meses el niño dominaría los dos primeros procesos supliendo la ausencia de la minimización formal por recursó a las formas diminutivas.

\section{Notas}

(1) Los números.

Los números se aprenden con gran facilidad pues todos se parecen a la realidad

el uno es un soldado haciendo la instrucción

el dos es un patito que está tomando el sol

el tres es una serpiente que baila sin cesar

el cuatro es una silla que invita a descansar

el cinco tiene orejas, parece un conejito

el seis es una pera redonda y con rabito

el siete es un sereno con gorra y con bastón

el ocho son las gafas que usa D. Ramón

el nueve es un globito atado a un cordel

el diez es un tiovivo para pasarlo bien.

(2) Para mejor comprensión del lector se transcriben las frases en lenguaje adulto. Naturalmente, el niño no las pronunciaba así en esta etapa. Para más detalles, ver Hernández-Pina (1984-a).

\section{Referencias}

Brace, A., y Nelson, D. L. (1965). The pre-school child's concept of number, The Arithmetic Teacher, $12,126-133$

BRUNER, J. S. (1964). The course of cognitive growth, American Psychologist, 19, 1-15.

BRUSH, L. R. et al. (1978). Children's difficulties on quantitative tasks: are they simply a misunderstanding of relational terms?, Joumal of Research in Mathematics Education, 9, 149-151.

CARSON, J. C. (1973). Parent power: primary activites for the home, University of Mississippi.

DODWEIL, P. C. (1960). Children's understanding of number and related concepts, Canadian Journal of Psychology, 14, 191-204.

DONALDSON, M., y BAIFOUR, G. (1968). Less is more: a study of language comprehension in children British Journal of Psycholingüistics, 59, 461-71.

DUTTON, W. H. (1983). Growth in number readiness in kindergarten children, The Arithmetic Teacher, May.

ELKILD, D. (1961). The development of quantitative thinking: a systematic replication of Piaget's studies. The Journal of Genetic Psychology, 98, 37-46.

ESTES, B. W. (1956). Some mathematical and logical concepts in children, The Jourmal of Genetic Psychology, 88, 219-22.

GiNSBERG, B. (1971). Mathematical concept learning by the pre-school child. Final Report. National Center for Educational Research and Development. California, San José State College.

HeRnández-Pina, F. (1979). Etapas en la adquisición del lenguaje: estudio de un caso concreto. Infancia y Aprendizaje, 8, 23-32.

HERNÁNDEZ-PINA, F. (1981). Adquisición del lenguaje infantil: etapa del balbuceo. Infancia y Aprendizaje, (Monografía n. 1 ), 71-84.

........ (1984a). Teorias psicosociolingüisstica y su aplicación a la adquisición del español como lengua matema. Madrid: Siglo XXI.

---.-- (1984b). Actitudes lingüisticas parentales y desartollo del lenguaje infantil, Infancia y Aprendizaje, 25, 35-60.

KING, M. L. y otros (1984). Language learning as learning how to mean: implications for practice from selected language research. Comunicación presentada en el Annual Meeting of the American Educational Research Association. 
KRANER, R. E. (1977). The acquisition age of quantitative concepts of children from three to six years old, Joumal of Experimental Education, 46, 52-59.

LIEBECK, P. (1984). How children learn mathematics. A guide for parents and teachers. Harmondsworth: Penguin Books.

MONROY, R., y HeRNÁNDEZ-PiNA, F. (1983). Un interrogante al esquema universalista de Jakobson

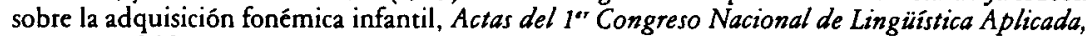
SGEL (Madrid), 143-156.

MuÑoz, C. (1984). Adquisición del castellano como segunda lengua. Estudio de un caso. Actas del II Congreso Nacional de Ling. Aplicada. (Granada), 231-44.

Novilus, C. F. (1979). The meaning of some relational and dimensional terms in children's lan. guage, Comunicación en el 6th Meeting of the National Conference on Diagnostic and Prescriptive Mathematics. Tampa, FL.

Piaget, J., e InHELDER, B. (1969). The psychology of the child. Nueva York: Basic Books.

ROBB, M., y LORD, C. (1981). Early uses of big and little by mothers and children. Papers and Re ports on Child Language Development, 20, 108-115.

REYS, R. E., y REA R. (1970). The comprehensive mathematics inventory: an experimental instrument for assessing youngsters entering school, Joumal of Research in Mathematics, 1, 180-86.

SCHWARTZ, A. N. (1969). Assessment of mathematical concepts of five-year-old children. Journal of Experimental Education, 37, 67-74.

SIGUAN, M. (Ed.) (1984). Estudios sobre la psicología del lenguaje infantil. Madrid: Pirámide.

SKEMP, R. R. (1982). the psychology of learning mathematics. Harmondsworth: Penguin Books.

SơTo, P. (1981). No es tu hija, es mi mamá: la adquisición de términos de parentesco, Infancia y Aprendizaje. Monografia, n.0 1, 49-6.

TREHUB, S. E., y ABRAMOVITCH, R. (1978). Less is not more: further observations on nonlinguistic strategies, Joumal of Experimental Child Psychology, 25, 160-67.

VILA, I. et al. (1984). Aspectos de la comunicación adulto-niño: el lenguaje de la madre, Actas del II. ${ }^{\circ}$ Congreso Nacional de Ling. Aplicada, Granada, 245-260.

WILuams, A. H. (1965). Mathematical concepts, skills and abilities of kindergarten entrants, The Arithmetic Teacher, 12, 261-68.

WINER. S. L. (1974). On the development of more and less, Joumal of Experimental Psychology, $17,277 \cdot 87$.

\section{Extended summary}

In this study we set out to analyse the influence of developing language in the learning of mathematics, a field of undeniable importance to parents and educationalists alike, focusing in particular on the field of quantification. Works on the subject of quantitatives as used by children are few and far between, particularly in Spanish. In the relevant foreign literature it is possible to discern four main strands in deâling with this topic. Some research studies have centered on the amount of quantitative terms children have by the age of six (Dutton, 1963; Brace y Nelson, 1965; Williams, 1965; Donalson y Balfour, 1968; Ginsberg, 1971; Brush y col, 1978, etc). Others endeavour to develop an instrument which could systematically assess the mathematical achievements of children aged two through five (Schwartz, 1969; Reys y Rea, 1970; Kraner, 1977, etc). To another group would belong those who are concerned about the role parents have in the development of quantitative language (Carson, 1973; King, 1984 , etc). Finally, there are those who try to see the extent to which quantitative adquisition validates Piaget's statements about children's language and thinking (1926 y 1929).

This article serves a dual purpose. On the one hand, an attempt is made to assess the acquisition of quantifiers and progress made by a Spanish-speaking child. On the other, some provisional guidelines are put forward for the development of quantitatives in Spanish. In order to explore this I have resorted to my son's speech, whose linguistic development I had previously traced from birth to the age of three (1984). Data gathering was made by using both taperecordings and day-to-day annotations of his free linguistic utterances. Within the latter, special attention was paid to the situational context. 
The results show the importance quantification seems to have in child's language. The grammatical distinction between plural and singular appears as the starting-point of quantification since it represents the splitting of the experience into a first and fundamental division: the unit versus the plurality of objects. The lack of plural forms during the holophrastic phase in Rafael's speech (from 18 to 24 months) would confirm the idea of a vague initial whole from which plurality would arise. During the two-word phase, numerals begin to emerge: although initially in a rather mechanic way, the child began to use UNO (one) both as a numeral and as an indefinite article by the age of 24 months. Two months later, the process of differentiation was well under way. DOS (two) as a concept preceded the acquisition of contable nouns as well as the term OTRO (other), the latter related to the degree of identity. Number TRES (three) emerged by the age of 37 months. There seems to be a striking parallelism between biological age and the acquisition of cardinal numbers. In contrast, PRIMERO (first) was the only term of the whole ordinal series used throughout the entire period ( 0 to 3 years). Within the distributive group, CADA UNO (each one) was regularly used from 31 months onwards.

One of the earliest and more sound acquisitions by Rafael was the use of the indefinite quantifier MAS (more), unlike the late appearance of MENOS (less). Surely this was due to the semantic load MAS has in the system. This is more noticeable in children's speech where this particle bears a high degree of suprageneralization: in the case of our informant it was used both as a quantifier and as an early substitute for assertive SI (yes). Possitive quantifiers would emerge much earlier than negative ones. Still within indefinite quantification we have another axis ranging from full to zero quantification $(\mathrm{TODO}(\mathrm{S})=$ all/everybody vs NADA = nothing/ NADIE (nobody). In between, intermediate quantification (ALGO = something) was rare, the child resorting to NADA instead. Of a later appearance (30 months) were the pairs ALGUNO/NINGUNO (any/no), ALGUIEN/NADIE (anyone/no-one). The early and versatile use of $\mathrm{MUCHO}(\mathrm{S})$ (little/few), thus supporting the view (Zipf) that positive terms are acquired earlier and are more numerous than their negative counterparts.

Finally, it is worth noticing the emergence of gradatives of comparison. They began to appear by the age of 22 months out of the equal-comparison structure. During the first stages, similarity and comparison went hand in hand. From 26 months onwards the alternative with COMO (similar) begins to consolidate to reach the stage, by the age of 43 months, in which there is a full mastery of the comparative degree forms IGUAL y COMO (same and similar). Equality in the sense of total identity was a reality by the three years of age. No trace, though, was found of structures involving non-equal comparisons of an ainferiority kind as exemplified by MENOS (less) (no doubt due to the low functional load of this particle, always substituted by other diminishers). Superlative forms were fairly common. Structures with - ISIMO (the very most...) made their appearance by the age of 30 months; during this time too our informant began to make use of quantification, either definite or indefinite (e.g. NUMERAL + VEZ-SIEMPRE/NUNCA). At the age of 41 months there appeared the periphrasis ASI DE (ever so...). Only numerals referring to duration did not turn up over the whole period. 